Review Article

\title{
Fetuin-A: A Biomarker for Type II Diabetes Mellitus
}

\section{Chukwunonso Livinus Udeh}

Department of Clinical Research, Amity Medical School, Amity University, Haryana, India

\section{Email address:}

lilnons2000@yahoo.com

\section{To cite this article:}

Chukwunonso Livinus Udeh. Fetuin-A: A Biomarker for Type II Diabetes Mellitus. International Journal of Biomedical Materials Research . Vol. 5, No. 6, 2017, pp. 72-77. doi: 10.11648/j.ijbmr.20170506.12

Received: October 30, 2017; Accepted: November 16, 2017; Published: December 27, 2017

\begin{abstract}
Fetuins are blood glycoproteins manufactured in the liver and secreted into the bloodstream. These glycoproteins make up a large group of binding proteins which mediates the transit and presence of a myriad of substances in the blood stream. Serum albumin, which is the most abundant protein in adult animal plasma is best known as the representative of these carrier proteins. In bone homeostasis, it is the circulating glycoprotein that plays a critical role. It plays major roles also in prevention of vascular calcification, disruption of adipocyte function and impairment of insulin signaling. Even with its major role, it is highly dependable in preventing and/or amplifying of the above disease processes. Certain diseases have been associated with high levels of Fetuin A. Although low levels of it in the plasma assist potentially in the protective effect of artery calcification in non-Chronic Kidney Disease (CKD) patients, high levels of the glycoprotein are the greatest concern in patients at higher risk of cardiovascular disease (CVD) and diabetes. The range of serum fetuin A in healthy adults is between $0.4 \mathrm{and} 1 \mathrm{mg} / \mathrm{ml} \mathrm{serum}$. The most studied function of fetuin $\mathrm{A}$ is mediated by the D1 domain. But the domain that binds with insulin receptor is yet to be known. The B chain consists of 27 amino acid residues which are distributed unevenly among the charged and neutral portion. Fetuins are proteins highly expressed in the liver blood plasma. They bear post translational modifications in proteolytic processing, phosphorylation, complex glycosylation and sulfation. The precussors of Fetuin A in humans are single-chained to the mature circulating double-chain form. In septicaemia and bovine, human fetuin A is perceptible to further proteolytic cleavage. Since the discovery of Fetuin A as a glycoprotein that inhibits vascular calcification in early 1990s, the biologic attributed roles has increased and still increasing exponentially. Apart from the roles it plays in type II diabetes mellitus and cardiovascular disease, other roles have been noted. Other effects and roles of Fetuin A are still being researched on.
\end{abstract}

Keywords: Fetuin A, Type II Diabetes Mellitus, Calcification, Atherosclerosis, Serum

\section{Introduction}

The name Fetuin is coined from "fetal". Fetuins are blood glycoproteins manufactured in the liver and secreted into the bloodstream. They are more abundant in the blood of a growing fetus. These glycoproteins were first described by Pedersen K. O. in 1944. These glycoproteins make up a large group of binding proteins which mediate the transit and presence of a myriad of substances in the blood stream. Serum albumin, which is the most abundant protein in adult animal plasma is the best known representative of these carrier proteins [1]. The three main family members of Fetuins are the a2-HS-glycoprotein (genetic symbol AHSG)/Fetuin A and Fetuin B. Over five decades ago, Fetuins were identified. It is a major component of the bovine fetal serum. The bovine
Fetuin comprises of 341 amino acid globular protein linked by six internal sulphide bonds, three N-linked and two O-linked chains of Oligosaccharides. This primary amino acid sequence with the position of the cysteine residues are duly conserved in sheep, rat, mouse, bovine, human Fetuins. Regulation of fetuin levels in the plasma is in the manner of a negative acute phase reactant [2].

Fetuin A is a liver-phosphorylated glycoprotein. In bone homeostasis, it is the circulating glycoprotein that plays a critical role. It plays major roles also in preventing vascular calcification, disruption of adipocyte function and impairment of insulin signaling. Even with this major role, it is highly dependable in preventing and/or amplifying the above disease processes. Certain diseases have been associated with high levels of Fetuin A. They include type- 2 
diabetes mellitus (T2DM), obesity and metabolic syndrome. There is a positive correlation between high myocardial infarction and stroke with increased Fetuin A levels. However, low levels of the glycoprotein have been associated with detrimental effects from beneficial healthy living. An example is the link between low circulating fetuin-A with the high mortality rates associated with patients with chronic kidney disease (CKD). Although low levels also have a potentially protective effect on artery calcification in non-CKD patients. It seems like high levels of the glycoprotein is the greatest concern in patients at higher risk of cardiovascular disease (CVD) and diabetes. Additionally, to this higher risk for women that haven't attained menopause and men of the similar age towards diabetes and CVD, post-menopausal women develop declined bone mineral density and elevated abdominal fat. The negativity and side effects of these can adversely affect the quality and period of life span in women. There is a report suggesting that the risk of Cardiovascular diseases, metabolic syndrome and insulin resistance rises substantially in post-menopausal women compared to pre-menopausal women. Decrease in production of the hormones oestrogen and progesterone by ovaries is also linked with increased insulin resistance, intra-abdominal fat and increase in inflammatory markers. All these are associated with cardiovascular diseases and/or metabolic syndrome [3].

Fetuin A is known to stabilize calcium-phosphate in a complex enabling it to be cleared by the phagocytic system. It is fundamentally manufactured in the liver and down regulated during the acute phase. In healthy adults, the range of serum Fetuin $A$ is between 0.4 and $1 \mathrm{mg} / \mathrm{ml}$ serum. This dominates the alpha- 2 band on serum electrophoresis. Gene knockout in mice was used to determine the function of Fetuin-A, in vivo. The animals developed a broad soft tissue calcification combined with, renal failure and myocardial infarction. Therefore, this glycoprotein is assumed to act as chaperon for minerals which primarily prevents calcification and pathological mineralization. Other functions include the modulation of TGF-beta activities and insulin signaling. Multiples activities in cell culture have been noted in crude preparation of Fetuin A which may be due to Fetuin A-bound molecules [4].

The glycoprotein also known as $\alpha-2$ Heremans-Schmid glycol-protein (AHSG) is among the superfamily of inhibitors of protease. It's also a major hepatic secretory protein in humans. It has multiple normal biological and pathological functions. Some of which include bone metabolism regulation, inhibition of vascular calcification, control of protease activity, insulin resistance, breast tumor cell proliferative signaling and keratinocyte migration. Fetuin A is a highly expressed glycoprotein in variety of tissues in a fetus, but in adults, it is mainly expressed only in the hepatic region. It functions as an endogenous ligand for the innate immune Toll-like receptor 4 (TLR4). It also inactivates the insulin receptor tyrosine kinase by binding to $\beta$-subunit of insulin receptor thereby modulating insulin signaling [5].

Structure
Fetuin A is a glycoprotein with maximized content of sugar moieties with residues of sialic acid. It has a range of molecular mass between $51 \mathrm{kDa}$ to $67 \mathrm{kDa}$, depending on the amount of carbohydrate [6]. Deutsch isolated and characterised Fetuin with trichloroacetate buffers by fractionation method in 1954. But the characterisation was heterogeneous at acidic $\mathrm{P}^{\mathrm{H}}$. In 1960, R. G. Spiro used low temperature ethanol fractionation to come by a homogenous version of the glycoprotein. This was done in the presence of $\mathrm{Ba}^{2+}$ and $\mathrm{Zn}^{2+}$ both by electrophoresis and ultracentrifugation. With the $\mathrm{P}^{\mathrm{H}}$ range of 1.1 to 11.2 and immunochemical criteria included. Purification of Fetuin by column chromatography yielded inactive protein. There are disagreements about the molecular weight of Fetuin. The values reported are 48,700 from Perdersen's sedimentation-diffusion, 45,000 from Deutsch's sedimentation-diffusion, 44,700 from Kay and Green's sedimentation-equilibrium, 44,600 from Kay and Marsh's light scattering and 48,400 from Spiro's sedimentation-diffusion.

The structure of Fetuin is well structurally detailed. Fisher et al plus Spiro and Spiro gave the complete analysis of the amino acid sequence. Both conclusions are close except for the values of threonine and arginine where there is deviation of both results by $30 \%$. The most noteworthy feature is the absence of methionine. Including sialic acid, anions becomes excess over cations; which is consistent with the low isoelectric point of Fetuin. The total peptide content ranges from $78 \%$ to $79 \%$. This protein portion consist of a single polypeptide chain.

The carbohydrate consists of 13 to 14 residues of sialic acid per mole, 20 hexose sugars (12 galactoses to 8 mannoses) and 18 hexosamines. Fetuin visualised contains about three equivalent heteropolysaccharide units. Each unit has a molecular weight of 3500. Spiro reported that the most likely composition of these units are four residues of sialic acid, four residues of galactose, three mannoses and six hexosamines. The terminal positions are occupied by the sialic acid with its carboxyl groups free. When antibodies react with Fetuin, they are directed towards the polypeptide portion of the molecule rather than the carbohydrate portion. This carbohydrate portion consist of three branched polysaccharide units. In both the $\mathrm{N}$-terminal and $\mathrm{C}$-terminal positions, one isoleucine residue per mole of protein is seen. The penultimate amino acid on the carboxyl-terminal portion of the chain is Serine. 12 half-cysteine residues are present as 6 intrachain disulphide bonds in each molecule [7]. Isolated Fetuin from fetal calf serum contains 3 carbohydrate units linked to serine and threonine residues on the peptide chain. These carbohydrate units are distinct from the 3 asparagine-linked heteropolysaccharides initially discovered about the protein [8].

Fetuin A gene in humans is centralized on chromosome 3q27. This chromosome is also mapped as the susceptible locus for MetS and type II DM. There is an association between several nucleotide polymorphisms, adipocyte insulin action, type 2 diabetes and dyslipidemia. Three chains are the components of the precursor proteins of Fetuin A but, only 
two chains; A and B encoded by a single mRNA transcript makes up the active protein. Chain $\mathrm{A}$ is longer and it comprises of two cystatin-like domains; D1 and D2. It's made up 282 amino acids residues, contains 29\% alpha helix, 24\% beta pleated sheet and $26 \%$ reverse turns. The function of
Fetuin A that is mostly studied is mediated by the D1 domain. But the domain that interacts with insulin receptor is yet to be known. The B chain consists of 27 amino acid residues. These residues are unevenly distributed among the charged and neutral portion $[9,10]$.

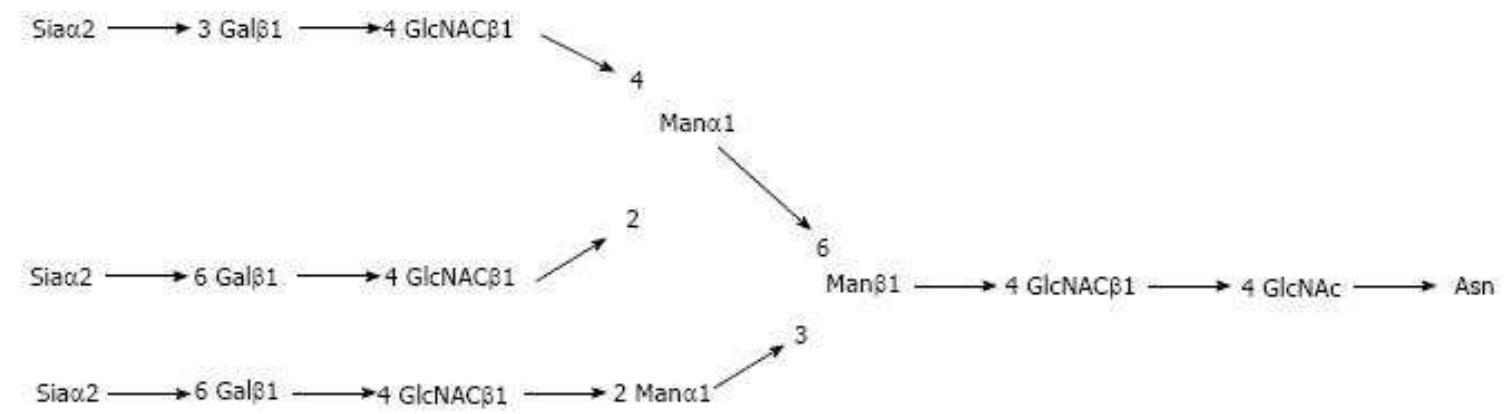

Figure 1. Structure of Fetuin A [11].

\section{Biosynthesis}

Fetuins are proteins highly expressed in the liver blood plasma. They bear post translational modifications proteolytic processing, phosphorylation, complex glycosylation and sulfation. The precursor of Fetuin $\mathrm{A}$ in humans are single-chained to the mature circulating double-chain form. In septicaemia and bovine, human Fetuin A is perceptible to enhance proteolytic cleavage. Procession of Fetuin A is by matrix metalloproteinases. Secondary modifications and allelic variants are seen in human Fetuin A/alpha 2-HS glycoprotein. Probably, these modifications assist in regulating the levels of protein expression, biological activity and stability. With insulin receptor, phosphorylation is indispensable whereas for mineral interaction, phosphorylation seems not to be required. Immediate hepatic clearing occurs through the asialogycoprotein receptor after Desialylation [12].

\section{Functions}

\subsection{Anti Inflammatory Role in Injury and Infection}

Acute phase proteins (APPs) are strategically synthesized and released by the liver in response to infection and injury. The concentration of these APPs are either increased (positive APPs) or decreased (negative APPs) in the plasma during inflammation. Fetuin A was initially characterized as an integral plasma protein in the fetus seen in many organs such as Liver, Gastro Intestinal Tract (GIT), Kidney, Skin, Brain, during fetal development. However, in adult humans, it is primarily produced by the Liver and annotates to divergently regulated synthesis in response to any injury of infection. It's classified as both negative and positive APP $[10,13]$.

The functions of Fetuin A are not yet properly understood despite its abundance in the blood plasma. Relying on its similarities in structure to other APPs and biogenic molecular physical interactions, a broader aspect of its functions biologically has been reported. Fetuin $\mathrm{A}$ is deciphered as an inhibitor of the TGF- $\beta$ signaling pathway because it shares an amino acid sequence homology to type II Transforming growth factor (TGF)- $\beta$ receptors. Fetuin A inactivates (rather than activating as seen in the case for insulin) the receptor tyrosine kinase because it exhibits amino acid sequence similar to those of insulin receptor tyrosine kinases. This may partially bring to understanding why patients with type II diabetes are known to be insulin resistant with high levels of Fetuin A [14,15]. The glycoprotein carries double N-linked and triple-linked oligosaccharide chains with sialic acid residues as it terminates, making it able to bind to $\mathrm{Ca}^{2+}$ ions. In soft tissues, Fetuin A inhibits pathological mineralization or calcification endogenously. It specifically forms protein-mineral colloids in combination with calcium and phosphate. Under pathological conditions, this helps to prevent uncontrolled mineralization [16]. As mentioned earlier, the glycoprotein also acts in cationic spermine as an opsonin and its presence in immune cells. This may be critical for regulation of the innate responses. Fetuin A reacts inversely when stimulated with Lipopolysaccharides (LPS; 100ng/ml, 2h). In macrophage cultures, stimulation with LPS reduced the levels of Fetuin A by $40 \%$. Supplementing LPS-stimulated macrophages with Fetuin A $(100 \mu \mathrm{g} / \mathrm{ml})$ conversely increased the cellular Fetuin-A levels by $30-50 \%$. This confirms the fact that macrophages can adopt Fetuin-A from their environment. Interestingly, Fetuin-A administered exogenously was predominantly localized in LC3-containing cytoplasmic vesicles; possibly, autophagosomes or amphisomes macrophages which are LPS-stimulated. Higher concentrations of even crude Fetuin A can almost completely repel endotoxin-induced nitric oxide release and IL-1 seen in macrophage cultures. From gel filtration and ion-exchange chromatography, highly purified Fetuin A almost completely evaded the release of IFN-y or LPS-induced HMGB1. Even when given at relative lower dose like $100 \mu \mathrm{g} / \mathrm{ml}$. This also suggest that Fetuin A is an effective anti-inflammatory APP [13]. 


\subsection{Fetuin A and Diabetes}

Fetuin $\mathrm{A}$ is in association with increased risk of being diabetic, most especially in individuals with high glucose in the plasma. This is because the glycoprotein inhibits the insulin-stimulated receptor tyrosine kinase, endogenously. Fetuin A administered to rodents caused the inhibition of insulin-stimulated tyrosine phosphorylation of the insulin receptor. Also the inhibition of insulin substrate 1 in skeletal muscles and liver of rat. Insulin sensitivity was experienced in Fetuin A knockout mice and there was resistance to the adipogenic effect of a high fat diet. This supports the hypothesis which says that $\alpha 2$-Heremans-Schmid glycoprotein, AHSG plays a pronounced role in the pathophysiology of insulin resistance in rodents. Norbert Stefan and his colleagues in 2008 showed high levels of circulating Fetuin-A is connected with resistance of insulin in humans. Other researchers have also proved the same effect suggesting that Fetuin-A may represent a novel mechanism in the pathophysiology of type II diabetes mellitus [17-20].

There is complexity in the pathophysiology of type II Diabetes Mellitus. In addition to the impairment of insulin secretion from beta cells in the liver, reduction in sensitivity of the hormone is also known to play a predominant role in the disease's pathogenesis. Both in in-vivo and in-vitro, Fetuin A is the secretory protein from hepatocytes that binds to insulin receptors and inhibit the glucose homeostasis regulation performed by insulin. Chromosome $3 \mathrm{q} 27$ is the region mapped out as a type II Diabetes Mellitus and metabolic syndrome susceptibility locus. Fetuin A is seen bound on this locus. Features of type II DM include inadequacy of insulin secretion and insulin resistance in the target tissues. Without phosphorylation of the insulin receptor, insulin won't be mediated. What Fetuin-A do is inhibit the autophosphorylation of this insulin receptor. Comparing lipid profile and its relationship with Fetuin-A, it has been observed that significant elevation in total cholesterol level, insignificant difference in serum triglycerides, LDL and HDL are all related to high Fetuin-A level. Such data suggests that Fetuin A is a marker associated with inflammation in both type II DM and obesity. In 2009, Baumann and his colleagues observed that Fetuin-A participates in inflammatory responses They stated that Fetuin-A increases in transcriptional events that leads to increase in expression of several pro-inflammatory cytokines including interleukins-1/6/12, and Tumor Necrosis Factor-A [21,22].

A study conducted on Chinese men and women concluded that concentrations of serum Fetuin-A are significantly higher in patients with type II DM than subjects within normal glucose regulation (NGR) and impaired glucose regulation (IGR). There was an independent association of higher concentrations of Fetuin-A with risk of insulin resistance not just in only non-diabetic subjects, but also in patients suffering from type II diabetes. The glycoprotein correlated positively with fasting serum insulin and HOMA-IR. Both indicates the activity of insulin resistance in type II DM [23].

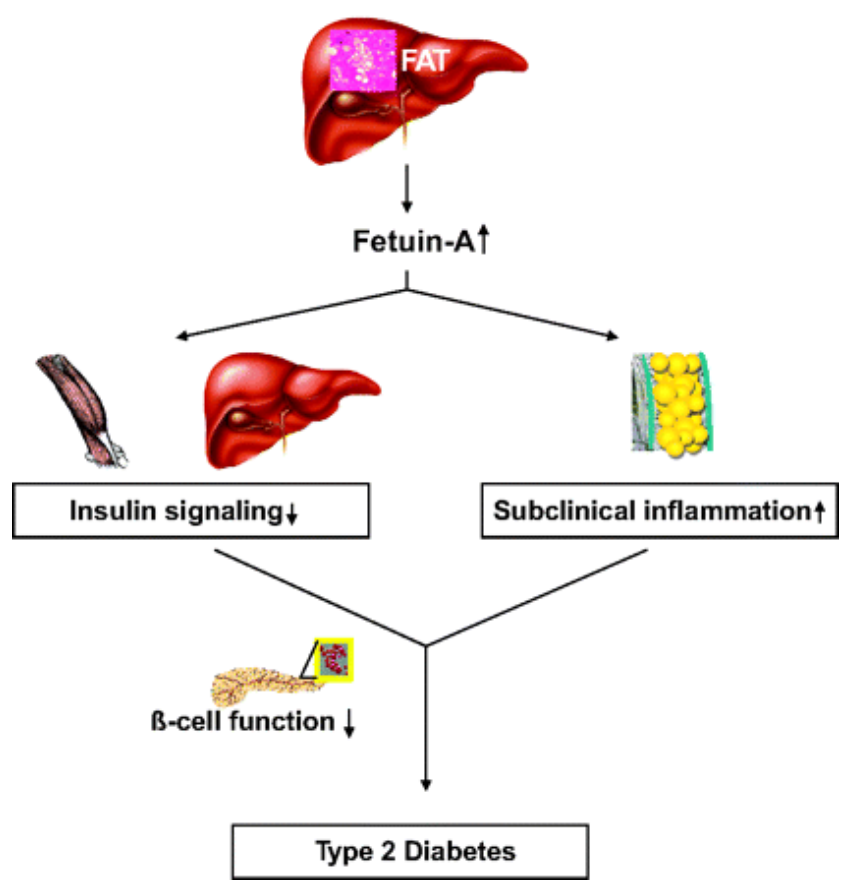

Figure 2. Schematic representation showing the effects of circulating plasma Fetuin-A on target tissues [20].

\subsection{Role of Fetuin-A in Cardiovascular Disease}

The role Fetuin-A plays in Cardiovascular diseases is more complex. It serves as a natural inhibitor of tissue and vascular calcification and also as a protective factor in Atheriosclerosis [24]. The first documented observational studies on Fetuin-A is the one conducted on patients with enhanced kidney disease. Lower concentrations of Fetuin-A were reported to be associated with risk of stroke and cardiovascular mortality. Moreover, more studies performed on general populations kept on generating mixed results. A study found no overall association, some reported positive associations and others, inverse associations. Two studies have indicated that elevated levels of Fetuin-A are associated with higher risk of cardiovascular diseases only in subjects suffering from insulin resistance. They concluded that that significant modification of Fetuin-A concentration in relation to cardiovascular diseases can be factored by Diabetes Mellitus and markers of Dysglycemia. These divergent findings are supported by the biological role of Fetuin-A in both insulin signaling and arterial calcification. From one angle, this glycoprotein forms colloidal complex with calcium and phosphorus. These complexes have the potential to reduce arterial calcification. From another angle, Fetuin A promotes the resistance of insulin by inhibition of the insulin receptor tyrosine kinase thereby mediating free fatty acid inflammatory signaling through toll-like receptor. There are competing actions of the role of Fetuin-A related to susceptibility of populations. It is likely to observe the role of Fetuin-A in populations in protection, with high prevalence of arterial calcification. This may explain why results were inverse and consistent, among patients with kidney disease. In the case of elevated glucose levels, Fetuin-A promotes insulin resistance and this may override its effects beneficially on inhibiting calcification. 
This also explains why there are positive associations of the glycoprotein among dysglycemic individuals in old studies [25-27].

\section{Future Aspects of Fetuin a Research}

Since the discovery of Fetuin A as a glycoprotein that inhibits vascular calcification in early 1990s, the biologic roles related to it has increased and still increasing exponentially. Apart from the roles it plays in type II diabetes mellitus and cardiovascular disease, other roles have been noted like; it acts as a negative acute-phase reactant in sepsis and endotoxemia, it promotes wound healing and has neuroprotective effect in patients with Alzheimer's disease. Also its low level in blood plasma is significant to increased disease activity in obstructive lung disease, ulcerative colitis and Crohn's disease. Other effects and role of Fetuin A is still being researched on.

\section{Discussion}

Fetuin A or $\alpha 2$-HS-glycoprotein is a part of the family of Fetuins secreted by the liver in adults. It is abundant in almost every tissue in the growing Fetus. In bone homeostasis, it is the circulating glycoprotein that play a critical role. It plays major roles also in prevention of vascular calcification, disruption of adipocyte function and impairment of insulin signaling. Even with these major roles, it is highly dependable in preventing and/or amplifying the process of the above diseases. Its precursor comprises of 3 chains; A, B, C. Chain A is longer and it comprises of two cystatin-like domains; D1 and D2. It's made up 282 amino acids residues, contains 29\% alpha helix, 24\% beta pleated sheet and $26 \%$ reverse turns. The function of Fetuin A that is mostly studied is mediated by the D1 domain. But the domain that interacts with insulin receptor is yet to be known. The B chain consists of 27 amino acid residues which are distributed unevenly among the charged and neutral portion. Fetuin A is classified as both negative and positive APP. It plays a role against inflammation and wound healing. The glycoprotein is also the hepatic secretory protein that binds to insulin receptors and inhibit the glucose homeostasis regulation performed by insulin. It serves as a biomarker for type II Diabetes Mellitus. In cardiovascular diseases, it serves as a natural inhibitor of tissue and vascular calcification and also as a protective factor in Atherosclerosis. Other negative and positive roles towards different diseases have also been noted.

\section{Conclusion}

The presence of Fetuin A in the plasma is of high significance in the prediction and diagnosis of many diseases ranging from Diabetes, Atherosclerosis, lung disease, ulcerative colitis and so on. It is a biomarker that cause both positive and negative effect. Its inhibition of insulin leads to glucose intolerance which can advance to elevation of fatty acid/lipids in the plasma, obesity and other cardiovascular diseases. Since the discovery of Fetuin A as an inhibitor of vascular calcification in early 1990s, and a glycoprotein known to have a high anti-inflammatory effect, the biologic roles attributed to it has and still increasing exponentially. There are many chances for advancements into its research.

\section{References}

[1] Fetuin [Internet]. [cited 2017 Oct 2]; Available from: https://en.wikipedia.org/wiki/Fetuin.

[2] Spiro RG. ARTICLE : a Glycoprotein of Fetal Serum *. J Biol Chem 1960; 225 (10): 2860-2869.

[3] Annie A, Peter W, Bane AA, Grandjean PW. The Role of Fetuin-A in Disease Processes Prevalent in Postmenopausal Women El papel de la fetuina A en los procesos de enfermedad prevalentes en mujeres posmenopáusicas. 2015;

[4] Häusler M, Schäfer C, Osterwinter C, Jahnen-Dechent W. The physiologic development of fetuin-a serum concentrations in children. Pediatr Res 2009; 66 (6): 660-664.

[5] Sindhu S, Akhter N, Shenouda S, Wilson A, Ahmad R. Plasma

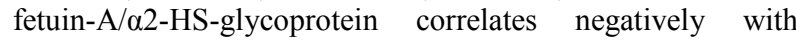
inflammatory cytokines, chemokines and activation biomarkers in individuals with type-2 diabetes. BMC Immunol [Internet] 2016; 17 (1): 33. Available from: $\mathrm{http}: / /$ bmcimmunol.biomedcentral.com/articles/10.1186/s1286 5-016-0171-y.

[6] Sakwe AM, Koumangoye R, Goodwin SJ, Ochieng J. Fetuin-A ( $\alpha 2 \mathrm{HS}$-glycoprotein) is a major serum adhesive protein that mediates growth signaling in breast tumor cells. $J$ Biol Chem 2010; 285 (53): 41827-41835.

[7] Neurath Hans. The Proteins: Composition, Structure, and Function. Volume III. Elsevier; 2012.

[8] Spiro RG, Bhoyroo VD. Structure of the O-Glvcosidicallv Linked Carbohydrates of Fetuin. J Biol Chem 1974; 249 (18): 5704-5717.

[9] Dabrowska AM, Tarach JS, Wojtysiak-Duma B, Duma D. Fetuin-A (AHSG) and its usefulness in clinical practice. Review of the literature. Biomed Pap 2015; 159 (3): 352-359.

[10] Singh M, Sharma PK, Garg VK, Mondal SC, Singh AK, Kumar N. Role of fetuin-A in atherosclerosis associated with diabetic patients. J Pharm Pharmacol 2012; 64 (12): 1703-1706.

[11] Eleni P., Despina S. P., Spiridon P. VSN. Role of fetuin A in the diagnosis and treatment of joint arthritis. World J Orthop [Internet] 2017; 8 (6): 461-464. Available from: https://www.wjgnet.com/2218-5836/full/v8/i6/WJO-8-461-g0 01.htm.

[12] Jahnen-Dechent W, Heiss A, Schäfer C, Ketteler M. Fetuin-A regulation of calcified matrix metabolism. Circ Res 2011; 108 (12): 1494-1509.

[13] Wang H, Sama AE. Anti-inflammatory role of fetuin-A in injury and infection. Curr Mol Med [Internet] 2012; 12 (5): 625-33. Available from:

http://www.pubmedcentral.nih.gov/articlerender.fcgi?artid=33 49766\&tool=pmcentrez\&rendertype $=$ abstract.

[14] Ix JH, Wassel-fyr C, Kanaya A, et al. NIH Public Access. 2009; 300 (2): 182-188. 
[15] Anton-Scott Goustin ABA-S. The "thrifty" gene encoding Ahsg/Fetuin-A meets the insulin receptor: Insights into the mechanism of insulin resistance. Cell Signal [Internet] 2011; 23 (6): 980-990. Available from: http://www.sciencedirect.com/science/article/pii/S0898656810 003153?via\%3Dihub.

[16] Rochette C. N, Rosenfeldt S., Heiss A, Narayanan T, Ballauff M J-DW. A shielding topology stabilizes the early stage protein-mineral complexes of fetuin-A and calcium phosphate: a time-resolved small-angle X-ray study. Chembiochem An Eur J Chem Biol 2009; 10 (4): 735-740.

[17] Sun Q, Cornelis MC, Manson JE, Hu FB. Plasma levels of fetuin-A and hepatic enzymes and risk of type 2 diabetes in women in the U.S. Diabetes 2013; 62 (1): 49-55.

[18] Mathews ST, Singh GP, Ranalletta M, et al. Improved insulin sensitivity and resistance to weight gain in mice null for the Ahsg gene. Diabetes 2002; 51 (8): 2450-2458.

[19] Laughlin GA, Barrett-Connor E, Cummins KM, Daniels LB, Wassel CL, Ix JH. Sex-specific association of fetuin-a with type 2 diabetes in older community-dwelling adults: The Rancho Bernardo study. Diabetes Care 2013; 36 (7): 1994 2000 .

[20] Stefan N, Fritsche A, Weikert C, Boeing H, Joost H, Schulze MB. Plasma Fetuin-A Levels and the Risk of Type 2 Diabetes. Diabetes 2008; 57 (10): 2762-2767.

[21] Baumann M, Richart T, Sollinger D, et al. Association between carotid diameter and the advanced glycation end product N-epsilon-carboxymethyllysine (CML). Cardiovasc Diabetol
[Internet] 2009; 8: 45. Available from: http://www.ncbi.nlm.nih.gov/entrez/query.fcgi?cmd=Retrieve $\& \mathrm{db}=$ PubMed\&dopt $=$ Citation\&list uids $=19660101 \% 5 \mathrm{Cnhttp}$ : //www.cardiab.com/content/pdf/1475-2840-8-45.pdf.

[22] Ahmed L, Khalaa MA-E, Mohamed NE-G, Mousa S, Yousry Z. Fetuin-A and type II diabetes mellitus. Egypt J Intern Med [Internet] 2014; 26 (4): 157. Available from: http://www.esim.eg.net/text.asp?2014/26/4/157/148140.

[23] Song A, Xu M, Bi Y, et al. Serum fetuin-A associates with type 2 diabetes and insulin resistance in Chinese adults. PLoS One 2011; 6 (4).

[24] Vörös K, Cseh K, Kalabay L. The role of fetuin-A in cardiovascular diseases. Orv Hetil [Internet] 2014; 155 (1): 16-23. Available from: http://www.akademiai.com/doi/abs/10.1556/OH.2014.29793.

[25] Chen HY, Lin CC, Chiu YL, et al. Serum fetuin A and chemerin levels correlate with hepatic steatosis and regional adiposity in maintenance hemodialysis patients. PLoS One 2012; 7 (7): 19.

[26] Laughlin GA, Cummins KM, Wassel CL, Daniels LB, Ix JH. The association of fetuin-A with cardiovascular disease mortality in older community-dwelling adults: The Rancho Bernardo study. J Am Coll Cardiol 2012; 59 (19): 1688-1696.

[27] Aroner SA, St-Jules DE, Mukamal KJ, et al. Fetuin-A, glycemic status, and risk of cardiovascular disease: The Multi-Ethnic Study of Atherosclerosis. Atherosclerosis [Internet] 2016; 248: 224-229. Available from: http://dx.doi.org/10.1016/j.atherosclerosis.2016.03.029. 